\title{
ON DERIVATIONS ANNIHILATING A MAXIMAL ABELIAN SUBALGEBRA
}

BY

\author{
GEOFFREY L. PRICE ${ }^{1}$
}

\begin{abstract}
Let $A$ be an AF $C^{*}$-algebra, and let $\delta$ be a closed *-derivation which annihilates the maximal abelian subalgebra $C$ of diagonal elements of $A$. Then we show that $\delta$ generates an approximately inner $C^{*}$-dynamics on $A$, and that $\delta$ is a commutative *-derivation. Any two closed *-derivations vanishing on $C$ are shown to be strongly commuting. More generally, if $\delta$ is a semiderivation on $A$ which vanishes on $C$, we prove that $\delta$ is a generator of a semigroup of strongly positive contractions of $A$.
\end{abstract}

1. Introduction. Let $\delta$ be a densely-defined $*$-derivation on a $C^{*}$-algebra $A$. One of the central problems in the theory of unbounded derivations is to determine whether a derivation is a generator, i.e., whether there exists a one-parameter group of $*$-automorphisms $\left\{\beta_{t}: t \in \mathbf{R}\right\}$ of $A$ such that for all $x \in D(\delta)$, the domain of $\delta$, $\delta(x)=\lim _{t \rightarrow 0}(1 / t)\left(\beta_{t}(x)-x\right)$, and such that $D(\delta)$ coincides with the set of all $x$ for which the limit above exists. Recently a number of articles have appeared considering this situation for the case where $\delta$ commutes with a group $\left\{\alpha_{g}: g \in G\right\}$ of automorphisms of $A$, i.e., $\alpha_{g}: D(\delta) \rightarrow D(\delta)$, all $g \in G$, and $\alpha_{g} \circ \delta=\delta \circ \alpha_{g}$. For example, Bratteli and Jørgensen have shown in [1] that a closed $*$-derivation must be a generator if it commutes with a compact abelian group of automorphisms of $A$ and annihilates the fixed point algebra $A^{\alpha}$ of $A$. Roughly speaking, their strategy is to decompose the algebra into spectral subspaces which are invariant under $G$ (and also $\delta$ ), to show that $\delta$ acts as a generator on each of these subspaces and to piece these results together to show that $\delta$ is a generator.

In this paper we consider an AF-algebra $A, A=\overline{U_{n} A_{n}}$, a certain maximal abelian $C^{*}$-subalgebra $C$ and a closed *-derivation $\delta$ which annihilates $C$. For $n \in \mathbf{N}$, we construct conditional expectations $\Phi_{n}$, mapping $\mathcal{A}$ onto the $C^{*}$-algebra $\left\langle A_{n}, C\right\rangle$ generated by $C$ and the finite-dimensional subalgebra $A_{n}$ of $A$. These maps are shown to respect the action of $\delta$ in the sense that $\Phi_{n}: D(\delta) \rightarrow D(\delta)$ and $\delta\left(\Phi_{n}(x)\right)=$ $\Phi_{n}(\delta x)$, all $x \in D(\delta)$. Using these techniques, we show that $\delta$ is a generator, and that any two derivations satisfying the above conditions are strongly commuting. Moreover, we exhibit a dense *-subalgebra of analytic elements of $\mathcal{A}$. We also show that $\delta$ is an approximately inner normal $*$-derivation which is commutative in the sense of Sakai [9]. Finally, we show that our techniques may be applied to prove analogous results in the more general case where $\delta$ is a $*$-semiderivation of $A$.

Received by the editors November 19, 1984.

1980 Mathematics Subject Classification. Primary 47B47.

Key words and phrases. Derivation, AF-algebra, maximal abelian subalgebra, approximately inner, generator.

${ }^{1}$ Supported in part by NSF Grant No. MCS- 8202290 . 
In a recent paper, $[\mathbf{1 1}], \mathrm{A}$. Kumjian uses somewhat different techniques to study similar questions on a class of $C^{*}$-algebras which includes the continuous trace AF-algebras. He shows that if $\delta$ is a closed *-derivation on a continuous trace AF-algebra which annihilates the diagonal subalgebra, then $\delta$ must be a generator [11, Theorem 5.2]. Moreover, a dense subalgebra of analytic elements for $\delta$ is also exhibited.

We have benefited from numerous comments and suggestions from F. Goodman, P. E. T. Jørgensen and R. T. Powers. It is a pleasure to thank them for their interest and help. We also thank G. Elliott for bringing [11] to our attention.

2. Diagonalization of an AF-algebra. In this section we introduce some notation which shall be needed in the proofs of our main results, and we also recall some facts and notation from [10] on the diagonalization of an AF-algebra. To begin, suppose that $A_{1} \subseteq A_{2} \subseteq \cdots$ is an ascending union of finite-dimensional $C^{*}$ algebras, with common identity 1 . Let $A$ be the unital AF-algebra formed as the uniform closure of the union $\bigcup_{n} A_{n}$. For any subsets $S_{1}, S_{2}, \ldots$ of $A$, let $\left\langle\bigcup_{n} S_{n}\right\rangle$ denote the smallest $C^{*}$-subalgebra of $A$ containing each of the $S_{n}$. In particular, $A=\left\langle\bigcup_{n} A_{n}\right\rangle$. Furthermore, if $B$ is a $C^{*}$-subalgebra of $A$, denote by $B^{\prime}$ the $C^{*}$ subalgebra of $A$ given by $\{x \in A: x y=y x$, all $y \in B\}$.

Following [10], we construct a maximal abelian $C^{*}$-subalgebra (m.a.s.a.) $C$, the diagonal subalgebra of $A$. $C$ is defined inductively as follows: let $A_{0}=\mathbf{C} \cdot \mathbf{1}$, and for each $n \in \mathbf{N} \cup\{0\}$, choose a m.a.s.a. $D_{n+1}$ of $A_{n}^{\prime} \cap A_{n+1}$. Set $C_{0}=\mathbf{C} \cdot \mathbf{1}$ and define $C_{n+1}=\left\langle C_{n}, D_{n+1}\right\rangle$. Then $C_{n}$ is a m.a.s.a. of $A_{n}$. Moreover, if $I$ and $J$ are index sets such that $\left\{p_{i}: i \in I\right\},\left\{q_{j}: j \in J\right\}$ are the minimal projections of $C_{n}, D_{n+1}$, respectively, then $\left\{p_{i} q_{j}: i \in I, j \in J\right\}$ is the set of minimal projections of $C_{n+1}$. A straightforward argument now shows that $C=\left\langle\bigcup_{n} C_{n}\right\rangle$ is a m.a.s.a. of $A$. More generally, one also obtains that, for fixed $n$, and for any $k \in \mathbf{N}$, the abelian $C^{*}$-algebra $A_{n}^{\prime} \cap C_{n+k}$ is a m.a.s.a. of $A_{n}^{\prime} \cap A_{n+k}$, and the uniform closure $\left\langle\bigcup_{k}\left(A_{n}^{\prime} \cap C_{n+k}\right)\right\rangle$ of the union is a m.a.s.a. of the AF-subalgebra $A_{n}^{\prime}$ of $A$. We refer the reader to the exposition in $[\mathbf{1 0}]$ for details.

In $[10]$ a conditional expectation $\Phi$ from $A$ to its m.a.s.a. $C$ is constructed. By conditional expectation it is meant that $\Phi: A \rightarrow C$ is a positive linear mapping which satisfies (i) $\Phi \circ \Phi=\Phi$, and (ii) $\Phi(x y)=\Phi(x) y$ and $\Phi(y x)=y \Phi(x)$ for $x \in \mathcal{A}$ and $y \in C$. In particular it follows that $\left.\Phi\right|_{C}$ is the identity mapping. These properties determine $\Phi$ uniquely. We now derive a slight generalization of this notion.

Proposition 2.1. Let $A=\overline{\bigcup_{n} A_{n}}$ be a unital AF-algebra. Then for any $n \in \mathbf{N} \cup\{0\}$ there exists a conditional expectation $\Phi_{n}$ from $A$ onto the $C^{*}$-subalgebra of $\mathcal{A}$ generated by $\mathcal{A}_{n}$ and the (diagonal) m.a.s.a. $C$ of $A$, i.e., $\Phi_{n}: \mathcal{A} \rightarrow\left\langle\mathcal{A}_{n}, C\right\rangle$.

Moreover, if $y \in \mathcal{A}_{n}^{\prime}$, then $\Phi_{n}(y) \in \mathcal{A}_{n}^{\prime} \cap \mathcal{C}$.

(REMARK. Observe that $\Phi_{0}$ coincides with the conditional expectation $\Phi$ described above.)

ProOF. Fix positive integers $m>n$. We begin by defining a conditional expectation $\Phi_{m, n}$ from $A$ onto $\left\langle A_{n}, A_{n}^{\prime} \cap C_{m}, A_{m}^{\prime}\right\rangle$. First recall (see, e.g., [5]) that for any $x \in A$ there exist elements $u_{i} \in A_{n}, v_{i} \in\left(A_{n}^{\prime} \cap A_{m}\right)$ and $w_{i} \in A_{m}^{\prime}$, $i=1,2, \ldots, r$, such that $x=\sum_{i=1}^{r} u_{i} v_{i} w_{i}$. Now let $\left\{p_{j}: j \in J\right\}$, some index set $J$, be the minimal projections of $A_{n}^{\prime} \cap C_{m}$. Consider the linear map $\Phi_{m, n}$ defined by 
$\Phi_{m, n}(x)=\sum_{j \in J} p_{j} x p_{j}$. Then

$$
\begin{aligned}
\Phi_{m, n}(x) & =\sum_{j \in J} \sum_{i=1}^{r} p_{j} u_{i} v_{i} w_{i} p_{j}=\sum_{j \in J} \sum_{i=1}^{r} u_{i}\left(p_{j} v_{i} p_{j}\right) w_{i} \\
& =\sum_{i=1}^{r} \sum_{j \in J} u_{i}\left(p_{j} v_{i} p_{j}\right) w_{i}=\sum_{i=1}^{r} u_{i}\left(\Phi_{m, n}\left(v_{i}\right)\right) w_{i} .
\end{aligned}
$$

Observe that for each $i, \Phi_{m, n}\left(v_{i}\right) \in A_{n}^{\prime} \cap \mathcal{A}_{m}$, and furthermore, $\Phi_{m, n}\left(v_{i}\right)$ commutes with $A_{n}^{\prime} \cap C_{m}$. But $A_{n}^{\prime} \cap C_{m}$ is maximal abelian in $A_{n}^{\prime} \cap C_{m}[\mathbf{1 0}]$, so that $\Phi_{m, n}\left(v_{i}\right) \in$ $\left(A_{n}^{\prime} \cap C_{m}\right)$. Hence $\Phi_{m, n}(x) \in\left\langle A_{n}, A_{n}^{\prime} \cap C_{m}, A_{m}^{\prime}\right\rangle$. Moreover, one checks easily that the latter algebra is fixed by $\Phi_{m, n}$, so that $\Phi_{m, n}$ is a conditional expectation onto $\left\langle A_{n}, A_{n}^{\prime} \cap C_{m}, A_{m}^{\prime}\right\rangle$.

It is straightforward to verify that for $x \in A_{r}$ and for $q>m \geq r, \Phi_{m, n}(x)=$ $\Phi_{q, n}(x)$. To see this, note that since

$$
A_{n}^{\prime} \cap C_{q}=A_{n}^{\prime} \cap\left\langle C_{m}, D_{m+1}, \ldots, D_{q}\right\rangle=\left\langle A_{n}^{\prime} \cap C_{m}, D_{m+1}, \ldots, D_{q}\right\rangle
$$

there exist minimal projections $e_{1}, \ldots, e_{s}$ in $\left\langle D_{m+1}, \ldots, D_{q}\right\rangle$ such that $\left\{p_{j} e_{k}: j \in\right.$ $J, 1 \leq k \leq s\}$ is the set of minimal projections of $A_{n}^{\prime} \cap C_{q}$. But since $e_{k} \in A_{r}^{\prime}$, all $k$, we have

$$
\begin{aligned}
\Phi_{q, n}(x) & =\sum_{j \in J} \sum_{k=1}^{s} p_{j} e_{k} x p_{j} e_{k}=\sum_{j \in J} \sum_{k=1}^{s} p_{j} x p_{j} e_{k} \\
& =\sum_{j \in J} p_{j} x p_{j}=\Phi_{m, n}(x) .
\end{aligned}
$$

Similarly, one may show that for $q \geq m \geq n, \Phi_{m, n} \circ \Phi_{q, n}=\Phi_{q, n}$.

We now use the results above to establish that for all $y \in A$, and for all fixed $n$, the sequence $\left\{\Phi_{m, n}(y): m>n\right\}$ converges uniformly. For, if $y \in A$, and $\varepsilon>0$, there exists an index $r$ and $x \in A_{r}$ such that $\|y-x\|<\varepsilon / 2$. Using $\left\|\Phi_{m, n}\right\|=1$, all $m>n$, we have for $q \geq m \geq r$,

$$
\begin{aligned}
\left\|\Phi_{q, n}(y)-\Phi_{m, n}(y)\right\| & \leq\left\|\Phi_{q, n}(y-x)\right\|+\left\|\Phi_{q, n}(x)-\Phi_{m, n}(x)\right\|+\left\|\Phi_{m, n}(y-x)\right\| \\
& \leq\|y-x\|+0+\|y-x\|<\varepsilon .
\end{aligned}
$$

Hence the sequence $\left\{\Phi_{m, n}(y): m>n\right\}$ has a uniform limit $\Phi_{n}(y)$. From the identity $\Phi_{m, n} \circ \Phi_{q, n}=\Phi_{q, n}, q \geq m \geq n$, it is straightforward to show that $\Phi_{m, n} \circ \Phi_{n}=\Phi_{n}$, and therefore, for all $y \in A$,

$$
\Phi_{n}(y) \in \bigcap_{m \geq n}\left\langle A_{n}, A_{n}^{\prime} \cap C_{m}, A_{m}^{\prime}\right\rangle
$$

We show $\left\langle A_{n}, C\right\rangle=\bigcap_{m \geq n}\left\langle A_{n}, A_{n}^{\prime} \cap C_{m}, A_{m}^{\prime}\right\rangle$. First, we have, for all $m \geq n$ and $q \geq n$,

$$
\left(A_{n}^{\prime} \cap C_{q}\right) \subseteq\left(A_{n}^{\prime} \cap C_{m}\right) \text { if } m \geq q
$$

and

$$
\begin{aligned}
\left(A_{n}^{\prime} \cap C_{q}\right) & =\left\langle A_{n}^{\prime} \cap C_{m}, D_{m+1}, \ldots, D_{q+1}\right\rangle \\
& \subseteq\left\langle A_{n}^{\prime} \cap C_{m}, A_{m}^{\prime}\right\rangle \text { if } m<q
\end{aligned}
$$


so that, in either case, we have $\left(A_{n}^{\prime} \cap C_{q}\right) \subseteq\left\langle\mathcal{A}_{n}, \mathcal{A}_{n}^{\prime} \cap C_{m}, A_{m}^{\prime}\right\rangle$, and therefore,

$$
\begin{aligned}
\left\langle A_{n}, C\right\rangle & \left.=\left\langle A_{n}, A_{n}^{\prime} \cap C\right\rangle=\left\langle A_{n}, \bigcup_{q>n}\left(A_{n}^{\prime} \cap C_{q}\right)\right\rangle \quad \text { (from }[\mathbf{1 0}]\right) \\
& \subseteq \bigcap_{m \geq n}\left\langle A_{n}, A_{n}^{\prime} \cap C_{m}, A_{m}^{\prime}\right\rangle .
\end{aligned}
$$

For the reverse inclusion, suppose $x \in\left\langle A_{n}^{\prime} \cap C_{m}, A_{m}^{\prime}\right\rangle$, all $m \geq n$. Then clearly $x \in A_{n}^{\prime}$ and $x \in\left(A_{n}^{\prime} \cap C_{m}\right)^{\prime}$, all $m$, hence $A_{n}^{\prime} \cap\left[\left(A_{n}^{\prime} \cap C\right)^{\prime}\right]$. But $A_{n}^{\prime} \cap C$ is maximal abelian in $A_{n}^{\prime}\left[10\right.$, Proposition I.1.3], so that $x \in A_{n}^{\prime} \cap \mathcal{C}$. The equality

$$
\bigcap_{m \geq n}\left\langle A_{n}, A_{n}^{\prime} \cap C_{m}, A_{m}^{\prime}\right\rangle=\left\langle A_{n}, A_{n}^{\prime} \cap C\right\rangle=\left\langle A_{n}, C\right\rangle
$$

now follows immediately. Therefore $\Phi_{n}$ is a conditional expectation from $A$ to $\left\langle A_{n}, C\right\rangle$, as asserted.

Finally, observe that if $y \in \mathcal{A}_{n}^{\prime}$, then for $m>n, \Phi_{m, n}(y)=\sum_{j \in J} p_{j} y p_{j}$ also lies in $A_{n}^{\prime}$. Since $\Phi_{n}(y)=\lim _{m} \Phi_{m, n}(y)$, the last statement of the proposition must hold.

3. Derivations annihilating $C$. We now apply the preceding results to show that any closed $*$-derivation $\delta$ on $A$ which vanishes on the diagonal m.a.s.a. $C$ must necessarily be a generator. Our techniques will enable us to construct explicitly a dense *-subalgebra of analytic elements for $D(\delta)$ (Proposition 3.2). Moreover, a direct application of our results shows that the set of closed $*$-derivations annihilating $C$ forms a family of strongly commuting generators (Corollary to Theorem $3.3)$.

LEMMA 3.1. Let $\delta$ be a closed $*$-derivation vanishing on $C$. For $n \in \mathbf{N} \cup\{0\}$, let $\Phi_{n}$ be the conditional expectation onto $\left\langle\mathcal{A}_{n}, C\right\rangle$ constructed in $\S 2$. Then for any $x \in D(\delta), \Phi_{n}(x)$ is also in $D(\delta)$ and $\delta\left(\Phi_{n}(x)\right)=\Phi_{n}(\delta x)$.

PROOF. For $m>n$, recall that $\Phi_{m, n}(x)=\sum_{j \in J} p_{j} x p_{j}$, where $\left\{p_{j}: j \in J\right\}$ is the set of minimal projections of $A_{n}^{\prime} \cap C_{m}$. Hence if $x \in D(\delta)$, then $\Phi_{m, n}(x) \in D(\delta)$, and

$$
\delta\left(\Phi_{m, n}(x)\right)=\sum_{j \in \mathcal{J}} \delta\left(p_{j} x p_{j}\right)=\sum_{j \in J} p_{j}(\delta x) p_{j}=\Phi_{m, n}(\delta x) .
$$

By Proposition 2.1, $\Phi_{n}(x)$ and $\Phi_{n}(\delta x)$ are the uniform limits of $\left\{\Phi_{m, n}(x): m>n\right\}$ and $\left\{\Phi_{m, n}(\delta x): m>n\right\}$. Combining this result with (1), we conclude from the closedness of $\delta$ that $\Phi_{n}(x) \in D(\delta)$ and that $\delta\left(\Phi_{n}(x)\right)=\Phi_{n}(\delta x)$.

Proposition 3.2. Let $\delta$ be a closed $*$-derivation vanishing on $C$. Then $D(\delta)$ contains a dense *-subalgebra of analytic elements. In fact, if $x \in \bigcup_{n} \mathcal{A}_{n}$, then $x$ is an analytic element for $\delta$.

ProOF. Fix $n \in \mathbf{N} \cup\{0\}$. Since $A_{n}$ is finite-dimensional, it is isomorphic to a direct sum $\sum_{k=1}^{p_{n}} \mathcal{M}_{r_{k}}$ of $r_{k} \times r_{k}$ matrix algebras $\mathcal{M}_{r_{k}}$ over $\mathbf{C}$. Hence, for $1 \leq k \leq p_{n}$, one may choose matrix units $e_{i j}^{k} \in A_{n}\left(1 \leq k \leq p_{n}, 1 \leq i, j \leq r_{k}\right)$ satisfying the identities (i) $e_{i j}^{k} e_{p q}^{k}=\delta_{j p} e_{i q}^{k}$, and (ii) for $k \neq l, e_{i j}^{k} e_{p q}^{l}=0$. Furthermore, we may assume that the matrix units have been chosen so that the diagonal elements $e_{i i}^{k}$ lie 
in $C$. To show that $A_{n}$ consists of analytic elements for $\delta$ it clearly suffices to show that the $e_{i j}^{k}$ are analytic.

We begin by showing that each matrix unit $e_{i j}^{k}$ lies in $D(\delta)$. For, if $1>\varepsilon>0$, there exists $x \in D(\delta)$ such that $\left\|x-e_{i j}^{k}\right\|<\varepsilon$. But then $e_{i i}^{k} x e_{j j}^{k} \in D(\delta)$ and we have

$$
\left\|e_{i i}^{k} x e_{j j}^{k}-e_{i j}^{k}\right\|=\left\|e_{i i}^{k}\left(x-e_{i j}^{k}\right) e_{j j}^{k}\right\| \leq\left\|x-e_{i j}^{k}\right\|<\varepsilon .
$$

Hence we may assume, without loss of generality, that $x=e_{i i}^{k} x e_{j j}^{k}$. Therefore, $x$ admits a decomposition $x=e_{i j}^{k} y$, where $y \in A_{n}^{\prime}$ (see [5]); whence $\Phi_{n}(x)=$ $\Phi_{n}\left(e_{i j}^{k} y\right)=e_{i j}^{k} \Phi_{n}(y)$. Let $d_{1}=\Phi_{n}(y)$; then $d_{1} \in\left(A_{n}^{\prime} \cap \mathcal{C}\right)$, from Proposition 2.1, so that

$$
\begin{aligned}
\left\|e_{j j}^{k} d_{1}-e_{j j}^{k}\right\| & =\left\|e_{j i}^{k}\left(e_{i j}^{k} d_{1}-e_{i j}^{k}\right)\right\| \leq\left\|e_{i j}^{k} d_{1}-e_{i j}^{k}\right\| \\
& =\left\|\Phi_{n}(x)-\Phi_{n}\left(e_{i j}^{k}\right)\right\| \leq\left\|x-e_{i j}^{k}\right\|<\varepsilon .
\end{aligned}
$$

Since $\varepsilon<1$, the inequality above implies that $d_{1}$ is invertible in the commutative $C^{*}$-subalgebra $e_{j j}^{k} C$, i.e., there exists $d_{2} \in e_{j j}^{k} C$ such that $d_{1} d_{2}=e_{j j}^{k}$. But $d_{2} \in$ $D(\delta), \Phi_{n}(x) \in D(\delta)$, so that $e_{i j}^{k}=\Phi_{n}(x) d_{2} \in D(\delta)$.

From Lemma 3.1,

$$
\delta\left(\Phi_{n}(x)\right)=\delta\left(\Phi_{n}\left(e_{i i}^{k} x e_{j j}^{k}\right)\right)=\delta\left(e_{i i}^{k} \Phi_{n}(x) e_{j j}^{k}\right)=e_{i i}^{k} \Phi_{n}(\delta x) e_{j j}^{k}
$$

Hence $\delta\left(\Phi_{n}(x)\right)$ has the form $\delta\left(\Phi_{n}(x)\right)=e_{i j}^{k} d_{3}, d_{3} \in A_{n}^{\prime} \cap C$. Then writing $d=d_{3} d_{2}$ $\left(\in A_{n}^{\prime} \cap \mathcal{C}\right)$,

$$
\delta\left(e_{i j}^{k}\right)=\delta\left[\Phi_{n}(x) d_{2}\right]=\left[\delta\left(\Phi_{n}(x)\right)\right] d_{2}=e_{i j}^{k} d .
$$

Iterating, we have, for any $r \in \mathbf{N}, \delta^{r}\left(e_{i j}^{k}\right)=e_{i j}^{k}\left(d^{r}\right)$, so that $e_{i j}^{k}$ is easily seen to be an analytic element of $D(\delta)$.

REMARK. We note that a somewhat similar analysis to the foregoing is carried out in [8], where $\delta$ is a closed *-derivation on a UHF algebra $A$ of Glimm type $n^{\infty}$. There it is shown that if $\delta$ vanishes on the natural embedding of $S(\infty)$ into $A$ $(S(\infty)$ is the group of finite permutations on countably many symbols) and satisfies $\tau \circ \delta=0$, where $\tau$ is the unique trace on $\mathcal{A}$, then $\delta$ admits an extension to a generator on $A$.

From the proof of the proposition it is clear that $\left\langle A_{n}, C\right\rangle \subseteq D(\delta)$, all $n \in \mathbf{N}$, and that $\delta:\left\langle A_{n}, C\right\rangle \rightarrow\left\langle A_{n}, C\right\rangle$. Indeed, for $1 \leq k \leq p_{n}$, let $d_{j}^{k}, 1 \leq j \leq r_{k}$, be the diagonal elements satisfying $\delta\left(e_{1 j}^{k}\right)=e_{1 j}^{k} d_{j}^{k}$. Consider the (skew-hermitian) element

$$
i h_{n}=\sum_{k=1}^{p_{n}} \sum_{j=1}^{r_{k}}\left(e_{j 1}^{k}\right) \delta\left(e_{1 j}^{k}\right) .
$$

Then we have the well-known identity $\left.\delta\right|_{\mathcal{A}_{n}}=\left.\operatorname{Ad}\left(i h_{n}\right)\right|_{\mathcal{A}_{n}}$, i.e., for $x \in \mathcal{A}_{n}, x=$ $\left[i h_{n}, x\right]=x\left(i h_{n}\right)-\left(i h_{n}\right) x($ see $[5])$. But

$$
\begin{aligned}
i h_{n} & =\sum_{k=1}^{p_{n}} \sum_{j=1}^{r_{k}}\left(e_{j 1}^{k}\right) \delta\left(e_{1 j}^{k}\right) \\
& =\sum_{k=1}^{p_{n}} \sum_{j=1}^{r_{k}} e_{j 1}^{k} e_{1 j}^{k}\left(d_{j}^{k}\right)=\sum_{k=1}^{p_{n}} \sum_{j=1}^{r_{k}} e_{j j}^{k} d_{j}^{k}
\end{aligned}
$$


so that $h_{n} \in C$. Extending to $\left\langle A_{n}, C\right\rangle$, it is straightforward to show that $\left.\delta\right|_{\left\langle A_{n}, C\right\rangle}=$ $\left.\operatorname{Ad}\left(i h_{n}\right)\right|_{\left\langle A_{n}, C\right\rangle}$ as well. From this identity it now follows easily that $\left.\delta\right|_{\left\langle A_{n}, C\right\rangle}$ is a generator, with corresponding one-parameter group $\left\{\alpha_{t}^{(n)}: t \in \mathbf{R}\right\}$ given by

$$
\alpha_{t}^{(n)}(x)=\exp \left(-i t h_{n}\right)(x) \exp \left(i t h_{n}\right), \quad x \in\left\langle\mathcal{A}_{n}, C\right\rangle .
$$

Applying [4, Theorem 3.2.51] one shows that the closure $\delta_{0}$ of the derivation $\left.\delta\right|_{\left[\cup_{n}\left\langle A_{n}, C\right\rangle\right]}$ is an (approximately inner) derivation on $\mathcal{A}$ with corresponding oneparameter group $\left\{\alpha_{t}: t \in \mathbf{R}\right\}$ satisfying

$$
\alpha_{t}(x)=\lim _{n} \exp \left(-i t h_{n}\right)(x) \exp \left(i t h_{n}\right), \quad x \in \mathcal{A} \text {. }
$$

We show that $\delta_{0}$ coincides with $\delta$. Clearly $D\left(\delta_{0}\right) \subseteq D(\delta)$. To verify the reverse inclusion let $x \in D(\delta)$. Then $x=\lim _{n} \Phi_{n}(x)$ and $\delta(x)=\lim _{n} \Phi_{n}(\delta x)=$ $\lim _{n} \delta\left(\Phi_{n}(x)\right)$. Since $\Phi_{n}(x) \in\left\langle A_{n}, C\right\rangle \subseteq D\left(\delta_{0}\right)$, the result holds. Hence we have the following.

THEOREM 3.3. Let $\delta$ be a closed *-derivation vanishing on the diagonal m.a.s.a. $C$ of $A$. Then $\delta$ is a generator. In fact there exists a sequence $\left(h_{n}\right) \subseteq C$ of hermitian operators such that $\delta$ generates the approximately inner dynamics $\left\{\alpha_{t}: t \in \mathbf{R}\right\}$, where $\alpha_{t}(x)=\lim _{n} \exp \left(-i t h_{n}\right)(x) \exp \left(i t h_{n}\right), x \in \mathcal{A}$.

REMARK. Since the sequence $\left(h_{n}\right)_{n \in \mathbf{N}}$ lies in $C$, the hermitian operators are mutually commuting. Hence $\delta$ is a commutative $*$-derivation in the sense of Sakai (see $[\mathbf{9}]$ ).

We recall the following notion.

DEFINITION 3.1. Let $\delta, \delta^{\prime}$ be generators on a $C^{*}$-algebra $A$. Then $\delta, \delta^{\prime}$ are said to be strongly commuting if their corresponding one-parameter groups $\left\{\alpha_{t}: t \in \mathbf{R}\right\}$, $\left\{\alpha_{t}^{\prime}: t \in \mathbf{R}\right\}$ satisfy $\alpha_{t} \circ \alpha_{t_{1}}^{\prime}=\alpha_{t_{1}}^{\prime} \circ \alpha_{t}, t, t_{1} \in \mathbf{R}$.

COROLLARY. The set of generators which vanish on the diagonal m.a.s.a. $\mathrm{C}$ of A form a family of strongly commuting derivations.

PROOF. Let $\delta, \delta^{\prime}$ be two such derivations, and let $\left\{\alpha_{t}\right\},\left\{\alpha_{t}^{\prime}\right\}$ be their corresponding one-parameter groups. Then from the preceding theorem there exist operators $h_{n}, h_{n}^{\prime} \in \mathcal{C}$ for $n \in \mathbf{N}$ such that

$$
\left.\alpha_{t}\right|_{\left\langle A_{n}, C\right\rangle}=\operatorname{Ad}\left(\exp \left(-i t h_{n}\right)\right),\left.\quad \alpha_{t}^{\prime}\right|_{\left\langle A_{n}, C\right\rangle}=\operatorname{Ad}\left(\exp \left(-i t h_{n}^{\prime}\right)\right) \text {. }
$$

Since $h_{n}, h_{n}^{\prime}$ commute it is straightforward to verify that $\alpha_{t}\left(\alpha_{t_{1}}^{\prime}(x)\right)=\alpha_{t_{1}}^{\prime}\left(\alpha_{t}(x)\right)$ for $x \in\left\langle A_{n}, C\right\rangle$ and $t, t_{1} \in \mathbf{R}$. The result now follows by continuity.

4. Applications to semiderivations. Let $A$ be a $C^{*}$-algebra. A linear operator $\delta: D(\delta) \rightarrow A$ is said to be a semiderivation (or, alternatively, a dissipation) if it satisfies the following properties:

(a) $D(\delta)$ is a uniformly dense *-subalgebra of $\mathcal{A}$,

(b) $\delta(x)^{*}=\delta\left(x^{*}\right)$, all $x \in D(\delta)$, and

(c) $\delta\left(x^{*} x\right) \geq \delta\left(x^{*}\right) x+x^{*}(\delta x)$ for $x \in D(\delta)$.

A central problem in the theory of semiderivations is to determine when $\delta$ is the generator of a strongly continuous one-parameter contraction semigroup $\left\{\alpha_{t}: t \in\right.$ $\left.\mathbf{R}_{+}\right\}$of positivity-preserving maps. For semiderivations vanishing on $C$ we have the following analogues to Theorem 3.3 and its corollary. (We thank P. E. T. Jørgensen for suggesting this extension of our original results.) 
THEOREM 4.1. Let $\delta$ be a closed semiderivation annihilating the m.a.s.a. C of $A$. Then $\delta$ is the generator of a strongly continuous one-parameter contraction semigroup $\left\{\alpha_{t}: t \in \mathbf{R}_{+}\right\}$of symmetric, strongly positive maps, i.e., $\alpha_{t}\left(x^{*}\right)=\alpha_{t}(x)^{*}$ and $\alpha_{t}\left(x^{*} x\right) \geq \alpha_{t}(x)^{*} \alpha_{t}(x)$ for all $x \in \mathcal{A}, t \in \mathbf{R}_{+}$.

ProOF. We preserve the notation of Proposition 3.2. Since $C \subseteq D(\delta)$ and $\left.\delta\right|_{C} \equiv 0$, we have, by [3, Lemma 1.1],

$$
\delta(x y)=(\delta x) y, \quad \delta(y x)=y(\delta x), \quad y \in C, x \in D(\delta) .
$$

Now using (2) we may employ the "averaging" argument of Lemma 3.1 to conclude that for all $n \in \mathbf{N} \cup\{0\}$ and $x \in D(\delta), \Phi_{n}(x) \in D(\delta)$ and $\delta\left[\Phi_{n}(x)\right]=\Phi_{n}(\delta x)$. This implies that $\left\langle A_{n}, C\right\rangle \subseteq D(\delta)$ for all $n$, and

$$
\delta:\left\langle A_{n}, C\right\rangle \rightarrow\left\langle A_{n}, C\right\rangle .
$$

We consider $\left.\delta\right|_{\left\langle A_{n}, C\right\rangle}$. Since $\delta$ is everywhere defined on $\left\langle A_{n}, C\right\rangle$ it follows from [6, Theorem 1] that $\left.\delta\right|_{\left\langle A_{n}, C\right\rangle}$ is both dissipative and bounded. In particular, $\left\langle A_{n}, C\right\rangle$ consists of analytic elements for $\delta$. Now, let $x \in\left\langle\mathcal{A}_{n}, C\right\rangle$. Then $x$ may be decomposed as

$$
x=\sum_{k=1}^{p_{n}} \sum_{i, j=1}^{r_{k}} e_{i j}^{k} c_{i j}^{k}, \quad c_{i j}^{k} \in C .
$$

Following the proof of Proposition 3.2, there exists for each matrix unit $e_{i j}^{k} \in \mathcal{A}_{n}$ an element $d_{i j}^{k} \in \mathcal{C}$ such that $\delta\left(e_{i j}^{k}\right)=e_{i j}^{k} d_{i j}^{k}$. Then using (2) repeatedly we have, for $c_{i j}^{k} \in C$ and $r \in \mathbf{N}$,

$$
\delta^{r}(x)=\sum_{k=1}^{p_{n}} \sum_{i, j=1}^{r_{k}}\left(e_{i j}^{k} c_{i j}^{k}\right)\left(d_{i j}^{k}\right)^{r} .
$$

Define $\delta_{0}$ to be the semiderivation $\delta_{0}=\left.\delta\right|_{D\left(\delta_{0}\right)}$, where $D\left(\delta_{0}\right)=\bigcup_{n} A_{n}$. Then $\delta_{0}$ is a dissipative semiderivation whose domain contains a dense *-subalgebra of analytic elements. Hence $\delta_{0}$ is closable, and its closure $\overline{\delta_{0}}$ is a generator of a contractive semigroup $\left\{\alpha_{t}: t \in \mathbf{R}_{+}\right\}$[2, Theorem 5]. Now applying an argument identical to the one in the last paragraph of the proof of Theorem 3.3, we conclude that $\overline{\delta_{0}}$ coincides with $\delta$.

We now consider the semigroup $\left\{\alpha_{t}\right\}$. Since $\delta(x)^{*}=\delta\left(x^{*}\right)$ for $x \in D(\delta)$, it follows immediately that $\alpha_{t}\left(x^{*}\right)=\alpha_{t}(x)^{*}$, all $x \in \mathcal{A}$. We show that the maps $\alpha_{t}$ are strongly positive. For $n \in \mathbf{N}$, consider any $x \in\left\langle A_{n}, C\right\rangle$. Then since $\delta$ is a bounded dissipative semiderivation on $\left\langle A_{n}, C\right\rangle$ with $\delta(1)=0$, it follows from [7, Corollary $3]$ that $\left\{\left.\alpha_{t}\right|_{\left\langle A_{n}, C\right\rangle}: t \in \mathbf{R}_{+}\right\}$is strongly continuous and that $\alpha_{t}\left(x^{*}\right) \alpha_{t}(x) \leq \alpha_{t}\left(x^{*} x\right)$. Using the strong continuity on $\left\langle\mathcal{A}_{n}, C\right\rangle$ for each $n \in \mathbf{N}$, it follows immediately that the semigroup of contractions is strongly continuous on $A$. Now, using continuity, it is straightforward to show that $\alpha_{t}\left(x^{*}\right) \alpha_{t}(x) \leq \alpha_{t}\left(x^{*} x\right)$, all $x \in \mathcal{A}$, so that the semigroup is strongly positive.

COROLlaRY. Let $\delta, \delta^{\prime}$ be any two semiderivations on $A$ which annihilate $C$. Then $\delta$ and $\delta^{\prime}$ are strongly commuting generators of contraction semigroups.

ProOF. From the theorem we deduce that $\delta$ (respectively, $\delta^{\prime}$ ) are the generators of the strongly continuous contractive semigroups $\left\{\alpha_{t}: t \in \mathbf{R}_{+}\right\}$(respectively, 
$\left.\left\{\alpha_{t}^{\prime}: t \in \mathbf{R}_{+}\right\}\right)$. Fix $n \in \mathbf{N}$, and consider $x \in\left\langle\mathcal{A}_{n}, C\right\rangle$. Then, by the theorem, $x$ is an analytic element for both $\delta$ and $\delta^{\prime}$, i.e.,

$$
\alpha_{t}(x)=\sum_{n=0}^{\infty}\left[(t \delta)^{r}(x)\right] /(n !) \quad\left(\text { respectively, } \alpha_{t}^{\prime}(x)=\sum_{n=0}^{\infty}\left[\left(t \delta^{\prime}\right)^{r}(x)\right] /(n !)\right) .
$$

Using the formula in (3) for $\delta^{r}(x), r \in \mathbf{N}$ (and a similar formula for $\left(\delta^{\prime}\right)^{s}(x)$, $s \in \mathbf{N})$, it is trivial to verify that $\left[\delta^{r} \circ\left(\delta^{\prime}\right)^{s}\right](x)=\left[\left(\delta^{\prime}\right)^{s} \circ(\delta)^{r}\right](x)$. But from this identity it follows immediately that for $t, t_{1} \in \mathbf{R}, \alpha_{t}\left(\alpha_{t_{1}}^{\prime}(x)\right)=\alpha_{t_{1}}^{\prime}\left(\alpha_{t}(x)\right)$. By continuity this equation holds for all $x \in \mathcal{A}$, so that $\delta, \delta^{\prime}$ are strongly commuting semiderivations.

\section{REFERENCES}

1. O. Bratteli and P. E. T. Jørgensen, Unbounded derivations tangential to compact groups of automorphisms, J. Funct. Anal. 48 (1982), 107-133.

2. __ Unbounded *-derivations and infinitesimal generators on operator algebras, Proc. Sympos. Pure Math., vol. 38, part 2, Amer. Math. Soc., Providence, R.I., 1980, pp. 353-365.

3. O. Bratteli, P. E. T. Jørgensen, A. Kishimoto and D. W. Robinson, A $C^{*}$-algebraic Schoenberg theorem, Australian National University, 1983, preprint.

4. O. Bratteli and D. W. Robinson, Operator algebras and quantum statistical mechanics, vol. I, Springer-Verlag, New York, 1979.

5. G. Elliott, Derivations of matroid $C^{*}$-algebras, Invent. Math. 9 (1970), 253-269.

6. A. Kishimoto, Dissipations and derivations, Comm. Math. Phys. 47 (1976), 25-32.

7. D. Evans and H. Hanche-Olsen, The generators of positive semigroups, J. Funct. Anal. 32 (1979), 207-212.

8. R. T. Powers and G. Price, Derivations vanishing on $S(\infty)$, Comm. Math. Phys. 84 (1982), 439-447.

9. S. Sakai, On commutative normal *-derivations, Comm. Math. Phys. 43 (1975), 39-40.

10. S. Stratila and D. Voiculescu, Representations of $A F$-algebras and of the group $U(\infty)$, Lecture Notes in Math., vol. 486, Springer-Verlag, Berlin and New York, 1975.

11. A. Kumjian, Preliminary algebras arising from local homeomorphisms, Math. Scand. 52 (1983).

Department of Mathematics, United States Naval ACademy, Annapolis, MARYLAND 21402 